\title{
Cutaneous Melanoma: Management of Melanoma Brain Metastases and Molecular Testing
}

\author{
Presented by Douglas B. Johnson, MD, MSCl; Susan M. Swetter, MD; April K.S. Salama, MD; and Evan Wuthrick, MD
}

\section{ABSTRACT}

Several advances in diagnosis and treatment of cutaneous melanoma were discussed at the NCCN 2021 Virtual Annual Conference. First, advances in immunotherapies and targeted agents have enhanced the role of systemic therapies in the up-front management of brain metastases in melanoma while improving survival. With dual-agent immune checkpoint inhibitors, more than half of patients with asymptomatic brain metastases that are not in high-risk anatomic areas of the brain respond to treatment, and these responses appear to be durable, sparing many patients from neurosurgery and/or stereotactic radiosurgery. In addition, molecular tests increasingly have implications for clinical decision-making in later-stage disease. The most important genetic mutation in melanoma is the BRAF V600 mutation, which can be found in approximately $40 \%$ to $50 \%$ of cutaneous melanomas.

Due to advances in immunotherapies and targeted agents, systemic therapies play an integral role in the up-front management of brain metastases in melanoma. At the same time, the choices for molecular testing have proliferated, particularly gene expression profiling for determination of metastatic risk. For patients with cutaneous melanoma, these tests may have prognostic value beyond AJCC melanoma stage but require further investigation regarding their role in stratifying surveillance and treatment decisions in patients with advanced disease.

During the NCCN 2021 Virtual Annual Conference, April K.S. Salama, MD, Associate Professor of Medicine, Duke Cancer Institute, and Evan Wuthrick, MD, Professor of Radiation Oncology, Moffitt Cancer Center, discussed the changing landscape of systemic therapies in melanoma and an approach to multidisciplinary care. Douglas B. Johnson, MD, MSCI, Associate Professor of Medicine, Vanderbilt-Ingram Cancer Center, and Susan M. Swetter, MD, Professor of Dermatology, Stanford Cancer Institute, discussed when to order molecular tests in cutaneous melanoma, the prognostic value of the results, and the implications for clinical decision-making.

\section{Dual-Agent Immunotherapy in Asymptomatic Patients}

According to Dr. Salama, many patients presenting with brain metastases will probably need both systemic therapy and local brain-directed therapy over the course of treatment.
Although activity with anti-PD-1 monotherapy has been seen, Dr. Salama noted, longer-term follow-up of pivotal studies has shown the effectiveness of dual checkpoint inhibition with ipilimumab and nivolumab in asymptomatic patients. This approach is based on data from the phase II CheckMate 204 study, which enrolled patients with metastatic melanoma and at least one measurable, nonirradiated brain metastasis (tumor diameter, $0.5-3 \mathrm{~cm}$ ) and no neurologic symptoms to receive nivolumab + ipilimumab followed by nivolumab maintenance. ${ }^{1}$ Study protocol allowed prior BRAF/MEK inhibitors, but this was largely an untreated patient population that had not received immune checkpoint inhibitors in the metastatic setting.

"We saw high intracranial response rates $>50 \%$, and these responses appeared to be durable," said Dr. Salama. "This was important because for the first time with immunotherapies we see that intracranial response rates appear to mirror the extracranial benefit."

Moreover, for a patient population with historically very poor prognosis, 18-month landmark survival was $75 \%$ in asymptomatic patients treated with dual checkpoint inhibitors.

"Prior to the era of effective systemic therapies, median overall survival was somewhere around 4 months in this patient population," said Dr. Salama. "This was a very important study for [patients with asymptomatic brain metastases]."

In a separate cohort of symptomatic patients, the response rate dropped to approximately $20 \%$ and progression-free survival was significantly lower. According 
to Dr. Salama, this highlights that up-front systemic therapy is "most likely better suited to patients with asymptomatic brain metastases."

Of note, patients did experience a high rate of grade 3 to 4 adverse events, and a significant percentage of patients did not complete all 4 doses of the induction regimen.

"This definitely changes the paradigm and hopefully works toward a future where not every patient will need to have brain surgery or stereotactic radiosurgery or any kind of local therapy," said Dr. Wuthrick. "However, I would not advocate for this approach in a very large brain metastasis. Patients with a metastasis $>3.5 \mathrm{~cm}$ probably need several local therapies, surgery, and postoperative radiation therapy (RT) to get control of their intracranial disease."

"I would also not advocate for this approach in highrisk anatomic areas of the brain, such as the brainstem, thalamus, or internal capsule," he added.

\section{Treatment After Disease Progression on Systemic Therapy}

Patients who experience disease progression on up-front systemic therapy pose unique challenges, particularly patients with both a high burden of extracranial disease and brain metastases. From a systemic standpoint, said Dr. Salama, targeted therapy makes sense as standard of care, but there are special considerations.

The combination of dabrafenib + trametinib has been shown to improve clinical outcomes in BRAF V600-mutant metastatic melanoma without brain metastases. In the phase II COMBI-MB trial, however, investigators explored the activity of dabrafenib + trametinib in active melanoma brain metastases. ${ }^{2}$

"Results of [COMBI-MB] showed a fairly high intracranial response rate that mirrors what we saw in phase III studies for patients who largely had just extracranial disease," said Dr. Salama. "We think that quick clinical improvements can occur with BRAF and MEK inhibitors in patients with brain metastases without local therapy. However, because the median duration of response of 6.5 months is shorter than what we see in extracranial disease, which is typically 11 to 12 months, I think it's important to think about local therapies."

Toxicity is a concern with the concurrent administration of dabrafenib + trametinib and RT. A literature review of 27 publications described potential increase in dermatologic, pulmonary, neurologic, hepatic, esophage$\mathrm{al}$, and bowel toxicity among patients receiving combined BRAF inhibitor and radiation treatment. ${ }^{3}$ Of note, 7 publications noted potential intracranial neurotoxicity.

"It's also important to note that toxicity seems to be associated with greater immune response, suggesting that there may be additive or even synergistic toxicity between the 2 agents," said Dr. Wuthrick. "We agree with the recommendations of ECOG: patients who are on BRAF/MEK inhibitor combination therapy should hold the BRAF inhibitors for 1 day before and at least 3 days after RT. When following those guidelines, we haven't seen any additional toxicities."

\section{Stereotactic Radiosurgery Versus Whole-Brain RT}

According to Dr. Wuthrick, radiation oncologists are increasingly trying to avoid whole-brain radiation, especially in melanoma. Stereotactic radiosurgery is much better tolerated, and it's a 1-day procedure versus several trips to the radiation center, he explained. What's more, patients receiving stereotactic radiosurgery do not lose their hair, do not experience the same level of fatigue as with whole-brain radiation, and can resume physical activities much sooner.

"A number of studies looking at whole-brain RT versus up-front stereotactic radiosurgery have clearly demonstrated that an up-front stereotactic radiosurgery approach benefits most patients," said Dr. Wuthrick, who noted that the exceptions are patients with a large number of brain metastases, miliary disease, or leptomeningeal disease. "For older patients, the benefit [of stereotactic radiosurgery] is even greater, as whole-brain RT does have an impact on upper-level cognitive function and short-term memory formation."

Ultimately, despite the complexity of these cases, Drs. Wuthrick and Salama underscored the improved survival that has come with advances in immunotherapy and local approaches like stereotactic radiosurgery.

"Patients with melanoma brain metastases do much better than you were told in medical school," he said. "Their lifespan is no longer measured in weeks, but rather in months and sometimes even in years. With dualagent immunotherapy drugs, two-thirds of patients with asymptomatic brain metastases will respond, and we can spare many from ever having to undergo neurosurgery or stereotactic radiosurgery."

\section{Molecular Testing for Cutaneous Melanoma: BRAF V600 Mutations}

From single gene tests to targeted next-generation sequencing to whole genome sequencing, the choices for molecular testing have proliferated over the years. The most important genetic mutation in melanoma is the BRAF V600 mutation, which can be found in approximately $40 \%$ to $50 \%$ of cutaneous melanomas $(80 \%-90 \%$ of these mutations are V600E, $15 \%$ are $\mathrm{V} 600 \mathrm{~K}$, and the remaining 5\% are other V600). Clinicians should wait until stage III or IV disease to test for this mutation, according to Dr. Johnson.

"There's little value in testing at resistance, as patients who develop resistance to BRAF and other MEK 
inhibitors often have a wide variety of resistance mechanisms that may differ even within tumors of the same patient," he said. "It's also important to note that BRAF V600 mutations tend to occur early in carcinogenesis. It's extremely unlikely that a patient will acquire $B R A F$ V600 mutations on therapy."

Next-generation sequencing tests are likely the most sensitive, but single-gene or multigene PCR platforms are also appropriate. BRAF V600E immunohistochemistry with the VE1 antibody also has "fairly good" sensitivity (89.2\%) and specificity (96.2\%), said Dr. Johnson, but it misses V600K and other less common mutations. ${ }^{4}$

Patients with resected stage III disease who were treated with 12 months of dabrafenib + trametinib in the adjuvant setting showed a dramatic improvement in relapse-free survival versus placebo. ${ }^{5}$

"It's debated whether BRAF/MEK inhibition or anti-PD-1 is a better treatment option in the adjuvant setting, but it's certainly clinically indicated to test for $B R A F$ mutations in the stage III setting, because dabrafenib + trametinib is a recommended and approved option," said Dr. Johnson.

Three different BRAF/MEK inhibitor combinations are approved in the metastatic setting. The combination of encorafenib + binimetinib, for example, demonstrated improved overall survival (median, 33.6 vs 16.9 months), progression-free survival (median, 14.9 vs 7.3 months), and overall response rate (64\% vs $41 \%$ ) compared with vemurafenib. $^{6}$

"There are very active options for patients with metastatic disease," said Dr. Johnson. "Most of these data are for patients with BRAF V600E mutations, but melanomas with $B R A F$ V600K mutations still have excellent response rate and activity."

"There are very active options for patients with metastatic disease," said Dr. Johnson. "Most of these data are for patients with $B R A F \mathrm{~V} 600 \mathrm{E}$ mutations, but melanomas with $B R A F$ V600K mutations still have excellent response rate and activity."

\section{BRAF Non-V600 Alterations}

Up to $5 \%$ of melanomas have BRAF non-V600 alterations, which can be a mix of exon 11, exon 15, and fusions, which are more common in spitzoid melanoma. Testing should be performed for stage IV disease and requires a larger assay, such as next-generation sequencing.

Although not nearly as much data are available for $B R A F$ non-V600 alterations, there are case series and reports of responses to MEK inhibitors, with the most evidence for fusions and mutations in L597 and K601. ${ }^{7-9}$ Retrospective and preclinical studies also suggest that BRAF/MEK inhibitor combination may have more activity than MEK inhibition alone.
"MEK inhibitor activity seems largely to be a melanoma-specific phenomenon," said Dr. Johnson. "We've seen $20 \%$ to $30 \%$ response rates in small trials in melanoma with MEK inhibitors versus just 3\% in nonselected tumors." ${ }^{10}$

\section{Immunotherapy Biomarkers}

Human cancers have a wide spectrum of numbers of mutations. For example, although pediatric cancers and different blood cancers often have very low and very infrequent mutations, cancers like melanoma, which are driven by ultraviolet light or other carcinogens, have perhaps the highest mutational load.

Retrospective data have shown that high tumor mutational burden is strongly correlated with progression-free survival and overall survival in patients with melanoma treated with anti-PD-1 therapy. ${ }^{11}$ However, Dr. Johnson noted that the subject of whether this should be used to guide therapy remains somewhat controversial. The immunotherapy pembrolizumab is approved for cancers with at least 10 mutations per megabase irrespective of cancer type.

Finally, PD-L1 expression is an important biomarker for a number of different cancers, but is not currently recommended for treatment decision-making for melanoma. One phase III study showed a trend toward higher response rates for anti-PD-1 monotherapy in patients with high PD-L1 expression (53\%), said Dr. Johnson, but there are still high response rates in patients with low expression (33\%).

"Patients with low PD-L1 seem to have more benefit overall to combination immunotherapy, whereas patients with high PD-L1 expression seem have similar outcomes when treated with either combination immunotherapy or single-agent immunotherapy," he continued. "However, this test is not something that we recommend routinely."

\section{Prognostic Molecular Tests for Clinical Decision-Making}

According to Dr. Swetter, one of the more controversial aspects of the NCCN Clinical Practice Guidelines in Oncology (NCCN Guidelines) for Melanoma ${ }^{12}$ is regarding the use of molecular tests for prognosis and risk of relapse in cutaneous melanoma.

Prognostic gene expression profiling (GEP) tests involve extraction of mRNA from formalin-fixed paraffinembedded sections through reverse transcriptase PCR on a selected panel of genes to determine a risk score. There are 3 commercially available prognostic GEP tests for melanoma: DecisionDx-Melanoma (31-gene GEP; Castle Biosciences, Inc.), MelaGenix (11-gene GEP; NeraCare $\mathrm{GmbH}$ ), and Merlin Assay (8-gene GEP; SkylineDx). 
There is currently prognostic uncertainty in melanoma. Approximately $3 \%$ to $5 \%$ of patients with minimally invasive (T1) melanoma will ultimately develop distant metastatic disease, yet there is no reliable predictive factor to identify these patients, who represent $20 \%$ to $30 \%$ of melanoma deaths due to the prevalence of thin melanoma. Sentinel lymph node biopsy (SLNB) identifies only $5 \%$ to $7 \%$ of patients with nodal metastasis from thin melanoma. ${ }^{13}$

"Identifying individuals from a molecular standpoint who will do poorly versus well would be important," said Dr. Swetter. "However, prognosis is not necessarily predictive of survival in the modern era of melanoma therapy."

Accurate prognostic GEP testing could improve staging accuracy, reduce the number of unnecessary SLNBs, and inform decisions on imaging and adjuvant therapy, she explained, but there are still several unknowns. Nevertheless, experts estimate that up to $10 \%$ of all invasive melanomas are being GEP-tested with commercially available platforms. The most common prognostic GEP test for cutaneous melanoma in the United States is DecisionDx-Melanoma.

Since 2018, the Centers for Medicare \& Medicaid Services has covered GEP testing for patients aged $>65$ years with thin melanoma (up to $2 \mathrm{~mm}$ ) and expanded that coverage in 2020 to include any melanoma with at least a $0.3-\mathrm{mm}$ threshold. However, GEP tests are not advocated in national melanoma guidelines (both NCCN and American Academy of Dermatology [AAD]). The 2021 NCCN Guidelines for Melanoma ${ }^{12}$ and the 2019 AAD melanoma guidelines ${ }^{14}$ cite insufficient evidence to recommend routine molecular profiling assessment for baseline prognostication and note that current GEP tests should not replace well-established staging techniques such as SLNB. The NCCN Guidelines state that use of GEP testing according to specific AJCC-8 melanoma stage will require further prospective investigation in large, contemporary datasets of unselected patients, with a national Melanoma Prevention Working Group consensus statement emphasizing the need for integration or comparison with established melanoma risk predictors and nomograms, including the AJCC-8 risk prediction tool in development. ${ }^{12,15}$

"More evidence is needed to support clinical utility," Dr. Swetter concluded. "Hopefully, prospective studies that are planned will help us address whether GEP testing provides additional independent prognostic information to available clinical pathologic factors, whether it can inform the need for SLNB, and/or whether it will help guide adjuvant therapy decision-making."

Disclosures: Dr. Johnson has disclosed receiving grant/research support from Bristol-Myers Squibb Company, and Incyte Corporation; serving as a scientific advisor for Bristol-Myers Squibb Company, Merck \& Co., Inc, and Novartis Pharmaceuticals Corporation; and receiving consulting fees from Catalyst Biopharma, lovance Biotherapeutics, Inc., Janssen Pharmaceutica Products, LP, and OncoSec Medical Incorporated. Dr. Salama has disclosed serving as a scientific adviser FOR lovance, Novartis Pharmaceuticals Corporation, Pfizer Inc., and Regeneron Pharmaceuticals, Inc. The remaining presenters have disclosed no relevant financial relationships.

Correspondence: Douglas B. Johnson, MD, MSCl, Vanderbilt-Ingram Cancer Center, 2220 Pierce Avenue, 777 Preston Research Building, Nashville, TN 37232. Email: douglas.b.johnson@vumc.org Susan M. Swetter, MD, Stanford Cancer Institute, Stanford Dermatology/Cutaneous Oncology, 900 Blake Wilbur Drive, W3045, MC: 5843, Stanford, CA 94305. Email: sswetter@stanford.edu; April K.S. Salama, MD, Duke Cancer Institute, DUMC 3198, Durham, NC 27710. Email: april.salama@duke.edu; and Evan Wuthrick, MD, Moffitt Cancer Center, 12902 Magnolia Drive, St. 1046, Tampa, FL 33618. Email: evan.wuthrick@moffitt.org

\section{References}

1. Tawbi HA, Forsyth PA, Algazi A, et al. Combined nivolumab and ipilimumab in melanoma metastatic to the brain. N Engl J Med 2018; 379:722-730.

2. Davies MA, Saiag P, Robert C, et al. Dabrafenib plus trametinib in patients with BRAF V600-mutant melanoma brain metastases (COMBI$\mathrm{MB})$ : a multicentre, multicohort, open-label, phase 2 trial. Lancet Oncol 2017;18:863-873.

3. Anker CJ, Grossmann KF, Atkins MB, et al. Avoiding severe toxicity from combined BRAF inhibitor and radiation treatment: consensus guidelines from the Eastern Cooperative Oncology Group. Int J Radiat Oncol Biol Phys 2016;95:632-646.

4. Long GV, Wilmott JS, Capper D, et al. Immunohistochemistry is highly sensitive and specific for the detection of V600E BRAF mutation in melanoma. Am J Surg Pathol 2013;37:61-65.

5. Dummer R, Hauschild A, Santinami M, et al. Five-year analysis of adjuvant dabrafenib plus trametinib in stage III melanoma. N Engl J Med 2020;383:1139-1148.

6. Dummer R, Ascierto PA, Gogas HJ, et al. Encorafenib plus binimetinib versus vemurafenib or encorafenib in patients with BRAF-mutant melanoma (COLUMBUS): a multicentre, open-label, randomised phase 3 trial. Lancet Oncol 2018;19:603-615.

7. Nebhan CA, Johnson DB, Sullivan RJ, et al. Efficacy and safety of trametinib in non-V600 BRAF mutant melanoma: a phase II study [published online April 16, 2021]. Oncologist doi: 10.1002/onco.13795.

8. Menzer C, Menzies AM, Carlino MS, et al. Targeted therapy in advanced melanoma with rare BRAF mutations. J Clin Oncol 2019;37:3142-3151.

9. Danker M, Lajoie M, Moldoveanu D, et al. Strategy for a subset of class II BRAF mutant melanomas. . Clin Cancer Res 2018;24:6483-6494.

10. Johnson DB, Zhao F, Noel M, et al. Trametinib activity in patients with solid tumors and lymphomas harboring BRAF non-V600 mutations or fusions: results from NCI-MATCH (EAY131). Clin Cancer Res 2020;26: 1812-1819.

11. Johnson DB, Frampton GM, Rioth MJ, et al. Targeted next generation sequencing identifies markers of response to PD-1 blockade. Cancer Immunol Res 2016;4:959-967.

12. Swetter SM, Thompson JA, Albertini MR, et al. NCCN Clinical Practice Guidelines in Oncology: Melanoma: Cutaneous. Version 2.2021. Accessed February 19, 2021. To view the most recent version, visit NCCN.org

13. Faries MB, Thompson JF, Cochran AJ, et al. Completion dissection or observation for sentinel-node metastasis in melanoma. N Engl J Med 2017;376:221-2222.

14. Swetter SM, Tsao H, Bichakjian CK, et al. Guidelines of care for the management of primary cutaneous melanoma. J Am Acad Dermatol 2019;80:208-250.

15. Grossman D, Okwundu N, Bartlett EK, et al. Prognostic gene expression profiling in cutaneous melanoma: identifying the knowledge gaps and assessing the clinical benefit. 2020;156:1004-1011. 